\title{
Experience with a dynamic inexpensive video-conferencing system for frozen section telepathology ${ }^{1}$
}

\author{
J.P.A. Baak ${ }^{\mathrm{a}, \mathrm{b}, *}$, P.J. van Diest ${ }^{\mathrm{a}}$ and G.A. Meijer ${ }^{\mathrm{a}}$

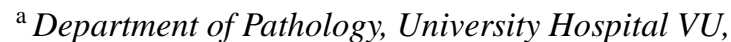 \\ Amsterdam, The Netherlands \\ ${ }^{\mathrm{b}}$ Department of Pathology, Medisch Centrum \\ Alkmaar, Alkmaar, The Netherlands
}

Aim: To evaluate the feasibility of an inexpensive, generally applicable video-conferencing system for frozen section telepathology (TP). Methods: A commercially widely available PC-based dynamic video-conferencing system (PictureTel LIVE, model PCS 100) has been evaluated, using two, four and six ISDN channels (128-384 kilobits per second (kbs)) bandwidths. 129 frozen sections have been analyzed which were classified by TP as benign, uncertain (the remark probably benign, or probably malignant was allowed), malignant, or not acceptable image quality. The TP results were compared with the original frozen section diagnosis and final paraffin diagnosis. Results: Only 384 kbs (3 ISDN-2 lines) resulted in acceptable speed and quality of microscope images, and synchronous image/speech transfer. In one of the frozen section cases $(0.7 \%)$, TP image quality was classified as not acceptable, leaving 128 frozen sections for the analysis. Five of these cases were uncertain by TP, and also deferred by frozen section procedure (FS). One more benign and three malignant FS cases were classified as uncertain by TP. Three additional cases were uncertain by FS, but benign according to TP (in agreement with the final diagnosis). In one case, FS diagnosis was uncertain but TP was malignant (in agreement with the final diagnosis). Thus, test efficiency (i.e., cases with complete agreement) was 120/128 $(93.8 \%$, Kappa $=0.88)$ between FS and TP. Sensitivity was $93.5 \%$, specificity $98.6 \%$, positive and negative predictive values were $97.7 \%$ and $96.0 \%$. Between TP and final diagnosis agreement was even higher. More importantly, there was not a single discrepancy as to benign-malignant. Moreover,

\footnotetext{
${ }^{1}$ Supported in part by grant \# 00-114 of the SBDM.

${ }^{*}$ Corresponding author: Prof. Dr. Jan P.A. Baak, MD, Ph.D., FRCPath, FIAC (Hon), Department of Pathology, Medical Center Alkmaar, Wilhelminalaan 12, 1815 JD Alkmaar, The Netherlands. E-mail: j.baak@mca.alkmaar.nl.
}

there was a clear learning effect: 5 of the 8 FS/TP discrepancies occurred in the first 42 cases $(5 / 42=11.9 \%)$, the remaining 3 in the following 86 cases $(3 / 86=3.5 \%)$. Discussion: The results are encouraging. However, TP evaluation is time-consuming (5-15 min for one case instead of 2-4 min although speed went up with more experience) and is more tiring. The system has the following technical drawbacks: no possibility to point at objects or areas of interest in the life image at the other end, resolution (rarely) may become suboptimal (blocky), storage of images evaluated (which is essential for legal reasons) is not easy and no direct control of a remote motorized microscope. Yet, all users were positive about the system both for telepathology and personal contact by video-conferencing. Conclusion: With a relatively simple videoconferencing system, accurate dynamic telepathology frozen section diagnosis can be obtained without false positive or negative results, although a limited number of uncertain cases will have to be accepted.

Keywords: Telepathology, frozen section, diagnosis, dynamic, synchronous

\section{Introduction}

Telepathology is the practice of pathology at a distance, viewing images on a video monitor rather than directly through a microscope. Excellent overview articles have been written on telepathology [20,22,23] also in the context of digital pathology [3]. Although the first telepathology tests were done already more than 30 years ago in the late sixties $[3,10]$, worldwide interest has grown rapidly over the past decade only [4, $5,8,9,12,15,17,19,21]$. No doubt, this is due to better and faster communication techniques, improved imaging methods, and also the penetration rate and acceptation of computer technology in pathology. As to the latter, better results with telepathology were obtained when the user had more video experience [21].

Typically, two different types of telepathology (TP) systems are discerned: static and dynamic. Static TP 
systems display still images [23], have no remote motorized control of the microscope at the other end, do not have life video-conferencing possibility and the network capacity (speed of communication) used typically (although not necessarily) is that of standard ana$\log$ telephone lines (up to 56,000 bits per second at best), using either a direct connection or an e-mail facility. Evaluation results are reasonably well $[2,11,16]$. The International Union Against Cancer (UICC) has decided to establish a static Internet-based Telepathology Consultation Center (UICC-TPCC) for interested pathologists around the world [7]. In this system, if a pathologist anywhere on the globe is confronted with the diagnosis of a difficult tumor case, he or she contacts the UICC-TPCC's World Wide Web server and uploads the images and the clinical data of their case. The center checks the case and transfers it to one of the UICC experts. This expert makes his or her diagnostic suggestion, which is then transferred back to the requesting pathologist via the UICC-TPCC.

On the other hand, questionnaires studies have shown that pathologists have a clear preference for dynamic TP systems with remote microscope control [14]. Indeed, dynamic TP systems have a number of advantages over static systems. Many thousands of life (real time) high quality images can be transmitted. The user can control, in the optimal case, a motorized microscope at the other end. Often, it is possible to see life images of persons at the other side or even have multiple-sites video-conferencing. However, health care worldwide requires economic TP systems $[1,6]$. Unfortunately, the communication channels used for dynamic telepathology systems are of the broad bandwidth type, and can be 2 megabits per second ( $2 \mathrm{Mbps}$ ) or higher. As a result, the running costs of such dynamic TP systems are still high. Moreover, top quality video-conferencing connections may be as expensive as USD 30,000 per month subscription rate. Dynamic telepathology systems are also much (three or more times) more expensive than static TP systems. Even although static TP systems can be obtained for USD 6,000, they are usually USD 15,000 and abovethus making dynamic TP not typically a bargain. Add to that the fairly limited applications (remote frozen section service, consultation at distance), and it does not seem very likely that apart from the UICC-TPCC system, current TP systems (static or dynamic) will quickly become extremely popular tools. Especially not in parts of the world that are fairly densely packed with adequate pathology services and good mail infrastructure - unless more economic systems will become available. Preferably, remote diagnostic video systems should have other functionalities than telepathology alone.

High-end PC-based real-time video-conferencing systems may neatly fall in this latter category. They consist of relatively inexpensive PC video-conferencing board/camera/phone/software packages, that can be installed on a standard Pentium PC, and a complete dynamic add-on videoconferencing package with adequate image quality may cost USD 4,000 to USD 10,000 . Add to that the costs of an adequate Pentiumbased PC and a reasonably priced complete videoconferencing system of USD 12,000 at the low end and USD 20,000 at the high end is coming in sight. Secondly, the gap between inexpensive (but slow) standard telephone lines and very expensive (fast) broad bandwidth communication lines could be filled by stacked $(2,3,4$ or more) ISDN-2 lines, which have an intermediate speed. ISDN-lines are now widely commercially available in many countries and hence not very expensive. One ISDN-2 line can carry 128 kilobits per second (kbs), and with 3- or 4-stacked ISDN-2 lines, transmission rates of up to 384 or $512 \mathrm{kbs}$ can be realized -5 to 14 times faster than a traditional analogue telephone line.

This article describes the experience with frozen section diagnosis using a commercially available, PC system for dynamic video-conferencing and telepathology.

\section{Material and methods}

\subsection{Equipment}

The PictureTel LIVE, model PCS 100 (PictureTel Corporation, Andover, MA, USA) has been evaluated. The system used (total price: USD 9,000) consisted of a Pentium $133 \mathrm{MHz}$ with $32 \mathrm{Mb}$ EDO memory and a $4 \mathrm{Mb}$ Matrox VRAM (video card), a Windows 95 operating system with mouse, camera, loudspeaker, microphone, and multiplexer to stack the different ISDN lines. The system works with the H320 standard for video-conferencing. The monitor has a VGA $(640 \times 480)$ resolution with a 24 bit color depth (true color). The transmitted image is of the so-called Full CIF (Common Interface Format (F-CIF)), format of $352 \times 288$ pixels, again in true color. Up to three cameras or video-recorders can be controlled by the system. A phonebook function is available. The camera and microphone can be switched off and on at both 
sites by clicking with the mouse on an icon. There are two subwindows, one for local video, and one for the remote signal, showing either the person on the other side of the line or the microscopic image or photograph. Each window can be resized, up to full screen or be minimized. A multiplexer can stack up to 3 ISDN2 lines (thus realizing $3 \times 128=384 \mathrm{kbs}$ transfer speed). The images are compressed before sending and decompressed at the other end, before displayed. Image and speech transfer speeds can be compromised to each other. Image quality speed can be improved at the expense of giving lower priority to speech transfer. After several tests, we gave first priority to image quality. This results in virtually instantaneous life image transfer, and hence very smooth displaying of moving images at the other end. Yet, although speech is given second priority, speech delay is less than 0.5 seconds. This is acceptable, although one has to get used to this slight delay.

\subsection{Cases}

We retrospectively analyzed 129 consecutive frozen sections and 9 additional standard other non-frozen section cases to explore the resolution limits of the system. One frozen section was regarded as too poor quality for reliable frozen section and TP evaluation. Thus, 138 cases remained for analysis (Table 1). The "telepathologist" got the usual information regarding the organ site, sex and age of the patient, as well as clinical data and the questions on the submission form. Each case was evaluated by TP without prior knowledge of the frozen section or definitive diagnosis, and could be classified as benign, uncertain (the additional remark probably benign or probably malignant was allowed), or malignant. Later, all diagnoses were compared with the original frozen section and final diagnoses. Discrepancy cases were reviewed by two independent pathologists, who both agreed with the definitive diagnosis. The overall agreement ("test efficiency") and the kappa statistics (to correct for chance) were calculated to compare the data.

We also calculated the sensitivity, specificity, positive and negative predictive values.

\section{Results}

Table 2 shows the evaluation by three observers (expert pathologists) of the different qualities of the system, at 128, 256 and 384 kbs. Only 3 stacked

\section{Table 1}

Specimens studied according to organ site. Between brackets the cases from which only standard paraffin sections (no frozen sections) have been studied

\begin{tabular}{lc}
\hline Breast & 52 \\
Cervix & $0(+3)$ \\
Colon & $4(+1)$ \\
Esophagus & 2 \\
Hypopharynx & 1 \\
Lip & 1 \\
Liver & 1 \\
Lung & 2 \\
Lymph nodes & 32 \\
Nasal mucosa & 3 \\
Omentum and peritoneum & 5 \\
Oral mucosa & 4 \\
Ovary & 10 \\
Parathyroid & 2 \\
Prostate & $3(+1)$ \\
Skin & $2(+1)$ \\
Stomach & $2(+2)$ \\
Testis & 1 \\
Uterus & 1 \\
Vagina and vulva & $1(+1)$ \\
Total & 138 \\
\hline
\end{tabular}

ISDN-2 lines (384 kbs) gave good results. The videoconferencing possibility of the 3 ISDN-2 system was scored as good to very good (on a scale of very bad to excellent) by 10 different independent observers. The multiple-site video-conferencing gave slower transmission of moving images, but still was acceptable for $\mathrm{H}+\mathrm{E}$ slides and also immunohistochemical (IHC) stains were easily interpretable. Mitoses, chromatin and nucleoli could well be discerned. In $\mathrm{H}+\mathrm{E}$ stains of the gastric mucosa, H. pylori could just not be visualized, whereas in specific IHC stains of consecutive sections, the $H$. pylori structures could be easily observed by TP.

Table 3 shows the confusion table of the definitive and TP diagnosis. The overall test efficiency (i.e., cases with complete agreement) was 120/128 (93.8\%, Kappa $=0.88$ ) between FS and TP. Sensitivity was $93.5 \%$, specificity $98.6 \%$, positive and negative predictive values were $97.7 \%$ and $96.0 \%$. Of the 8 disagreement cases, 5 occurred in the first 42 cases (discrepancy $5 / 42=11.9 \%$ ), whereas in the following 86 cases discrepancy occurred in 3 more cases only $(3.5 \%)$. Thus, there was a significant learning effect. No benign/malignant discrepancies occurred; most discrepancies were uncertain by TP, and either 
Table 2

Evaluation by ten independent observers (expert pathologists) of the different qualities of the video-conferencing-telepathology system

\begin{tabular}{l}
\hline 28 kbs (1 ISDN): \\
Blocky images \\
Serious image delay \\
Images/speech dissociation \\
Colours good \\
Overall unacceptable \\
256 kbs (2 ISDN): \\
Image quality suboptimal \\
Image delay \\
Speech delay unacceptable \\
Colours good \\
Overall: suboptimal \\
384 kbs (3 ISDN): \\
Image movement good, no delay \\
Sometimes somewhat blocky images \\
Speech delay: <0.5 sec, acceptable \\
Colours good \\
Overall: good
\end{tabular}

Table 3

Correlation between frozen section (FS) and telepathology (TP) diagnoses

\begin{tabular}{lcccr}
\hline & TP, benign & TP, uncertain & TP, malignant & Total \\
\hline FS, benign & 72 & $1^{(1)}$ & 0 & 73 \\
FS, uncertain & $3^{(2)}$ & 5 & $1^{(3)}$ & 9 \\
FS, malignant & 0 & $3^{(4)}$ & 43 & 46 \\
Total & 75 & 9 & 44 & 128 \\
\hline
\end{tabular}

Overall agreement: $(72+5+43) / 128=93.8 \%($ kappa $=0.88)$. Sensitivity $=93.5 \%$, specificity $=98.6 \%$, positive predictive value $=97.7 \%$, negative predictive value $=96.0 \%$.

(1) Ovary. Benign cystadenoma with tangential cutting. (2) (a) Benign liver nodule, (b) omentum, endosalpingiosis, (c) omentum, endosalpingiosis. (3) Breast. Invasive lobular carcinoma. (4) (a) Breast. Invasive lobular carcinoma, (b) breast. Tubular carcinoma, (c) Vagina. Adenocarcinoma.

benign or malignant by definitive diagnosis. Moreover, in all of these $3 \mathrm{TP}=$ uncertain discrepancies, the additional remark "uncertain, but probably benign" (in case of a benign mucinous cystadenoma of the ovary) or "uncertain, probably malignant" (in a case of lobular invasive Indian filing type breast cancer and one more case of tubular carcinoma of the breast) was added during the TP session. Thus, the discrepancies were marginal and clinically within the acceptable limits. In three cases, FS was uncertain and TP was benign (in agreement with the definitive diagnosis). In one case, FS diagnosis was uncertain and TP diagnosis was malignant (in agreement with the final diagnosis). This was a case of invasive lobular carcinoma of the breast, where the FS pathologist was junior and felt some uncertainty as to the diagnosis, whereas the TP diagnosis was made by one of us with considerable experience in breast pathology.

\section{Discussion}

The overall working experience with the PC-based video-conferencing system for frozen sections in a large pathology practice was positive, both for (multiple sites) video-conferencing and telepathology. Certainly, telepathology by PC-based videoconferencing does not solve all problems in the evaluation of frozen sections, but it can be helpful. Even if expert pathologists that are consulted by TP are not able to give a definitive answer, they may be able to give suggestions as to possible differential diagnoses, additional stains or laboratory and clinical tests required and thus still help the person at the other end.

In a recent economics analysis, Agha et al. [1] found that telepathology is less expensive than onsite pathology in a remote hospital. However, couriertype consultation on the other hand was less expensive than telepathology consultation. They therefore have argued that if the costs of telecommunication and telepathology systems could be lowered to reflect the current costs of the courier type consultation, then telepathology becomes the favored option for remote hospitals. In agreement with this demand, the price-performance ratio of the system evaluated in the present study is high. It is slightly more expensive (USD 14,000) than some, but not all, commercially available static telepathology systems (range: USD 6,000-35,000, excluding microscopes). However, the dynamic functionality with live images of the present video-conferencing system for telepathology is to be preferred. The possibility for multiple sites videoconferencing is a further advantage and quickly would make PC videoconferencing economic in a pathology laboratory. For example, urgent meetings by participants at more than 2 distant locations may not be possible at all, because of incompatibility of different agendas. Moreover, in such meetings the traveling time (easily 3 hours even in densely populated areas) for each of more than 3 participants makes PC based videoconferencing easily cost-effective. Multiple sites video-conferencing even can become essential in such situations. 
The agreement between TP, and definitive diagnoses was in the range, although at the high-end, of what has been published so far [3,20,22]. The material studied consisted of consecutive frozen sections in two large pathology laboratories and was thus unselected. There were, however, relatively many benign breast lesions that were easy to diagnose. In addition, the three pathologists involved in the evaluation all fall in the category of much video/PC experience.

There were 8 discrepancies between the TP and conventional frozen section diagnoses in the present series of 128 cases. However, no benign-malignant discrepancies occurred: all discrepancies were of the benign (definitive diagnosis) versus uncertain, or malignant (definitive diagnosis) versus uncertain type. Moreover, in the uncertain TP diagnoses often there were additional remarks made like: "uncertain, probably benign" (in case of a benign mucinous cystadenoma of the ovary) or uncertain, probably malignant (in a lobular invasive Indian filing type of breast carcinoma). As to the latter, we learned that the telepathology system has its own, characteristic diagnostic clues: initially, we found the lobular invasive parts difficult to distinguish from infiltration by leukocytes. However, by over-lighting the image, we later found that leukocytic infiltrates are much earlier "overlighted" (and thus disappear earlier on the video monitor) than infiltrating cancer cells. It is thus possible that in the future, as we learn more such typical TP characteristics, the efficiency of telepathology still will improve.

In spite of all the above-mentioned advantages, the PC-based dynamic telepathology system used also has serious disadvantages over both static and classical dynamic telepathology systems. Compared with static systems both the price and running costs of dynamic TP systems may be considerably higher. Inexpensive static telepathology system can be obtained for USD 6,000 (although most commercial systems are USD 15,000-30,000). Moreover, standard telephone lines that can be used for static, but not for dynamic telepathology, are cheaper than 3-stacked ISDN2 lines: in the Netherlands, annual subscription rates alone are USD 135 for one standard telephone line versus USD 750 for three combined ISDN lines (at a rate of $1 \mathrm{ECU}=0.84 \mathrm{USD}$, or $1 \mathrm{USD}=2.60 \mathrm{HFL}$ ). Based on our experience so far, the running costs for national "telepathology calls" would be USD 1,000 versus USD 4,500 per year. On the other hand, in the latter figure is included USD 1,000 for 4 multiple sites conferencing meetings, each of which was "attended" by 8 persons. A multiple sites conferencing meeting took no longer than 75 minutes or 6 person hours, whereas a standard meeting would have taken 24 working and traveling hours for one person per laboratory. The consequent saving of 18 person hours per meeting, times 4 meetings $=72$ working hours, plus traveling costs, would thus have exceeded greatly the total annual costs for calls of the 3-ISDN-2 video-conferencing system.

Two other disadvantages are related to each other. Working with the telepathology system takes more time than the "pure" time for a frozen section (5-15 min versus $2-5 \mathrm{~min}$ ). However, when frozen sections are done at a remote place, the traveling time normally exceeds this by far. Moreover, when working experience with the system increased, evaluation time by the pathologist dropped. Dunn et al. in an analysis of 2200 cases also experienced a considerable decrease in the pathologist's viewing time with increasing experience [8]. Furthermore, working with the telepathology system is tiring ("very intensive"), but especially so in the beginning. This has several reasons. First, a standard PC video image does not have the same high resolution as a microscope. As a result, more frequent changing to a higher objective is required, when scanning through a section, "just to be sure". Secondly, the system used did not have a bi-directional arrow in the life image; only the side sending the microscope image could use the usual microscope arrow pointer. In the frozen mode, bi-directional arrows are available. However, to do this the following actions have to be taken: saving the life image in the local life video mode, exporting to a subdirectory, re-importing it in a whiteboard window, opening and wait until both sides see the static (frozen) saved and re-opened image. This takes 40-60 seconds; too time-consuming to be a standard procedure.

A further disadvantage, both of the telepathology and video-conferencing mode of the system analyzed, is that it does not allow at the moment to control remote motorized microscopes or cameras (left-right, up-down, light intensity, condensor in-out, objectives change, etc.). Also not available is the possibility to switch from fast scan-standard F-CIF intermediate low resolution to slow scan-high resolution. It would be a very easy tool, if one could switch quickly, to be able to better see details of areas of doubt or special interest. This could save switching to a higher magnification, which occurs fairly often when scanning through a microscope slide. Also, zooming in and out, or leftright/up-down movement control of a camera during a video-conferencing session would be a very easy option to have. Finally, storage of the images is essential 
for telepathology diagnosis for legal reasons. This is possible with the current system but in a fairly primitive and still unsatisfactory manner.

In spite of these drawbacks, all users so far are positive about the system both for dynamic telepathology and video-conferencing. This is in agreement with recent findings by others. At $384 \mathrm{kbs}$, the image and speech quality is acceptable. One important advantage of the use of the video-conferencing system was that different people learned to know each other without ever having met "physically". As a result, two laboratories may function as a unity, to a certain extent; exchange of personnel becomes easier. Finally, the favorable price-performance ratio allows for a certain degree of telepathology, which otherwise would not have been possible, either by financial or quality restrictions.

In this article we have evaluated a telepathology system with a full synchronous connection. As to the differentiation between static and dynamic TP systems, technical developments over the past two years have shown that both strategies may be useful. This explains the fact that in some countries many static, in other countries pure dynamic systems prevail. Hybrid systems have been developed like static systems with remote control of microscopes. It thus may be better to discern in the future "synchronous" and "asynchronous" systems. Clearly, the system we have evaluated and described here falls in the synchronous category.

Next to the minor drawbacks we have mentioned, one could ask whether sampling errors are a major problem in telepathology. Of course, this cannot be completely excluded, but a recent study showed that this is certainly not a major problem [5]. Moreover, in a recent review article, Wells and Sowter [26] have summarized the enormous developments in telepathology over the past 20 years and also indicated the two residual problems. In their opinion, the first concerns the speed of data transmission. In view of the present results obtained with standard ISDN telephone lines, we do not quite agree with this. The second problem they mention is that the software provided by most of the manufacturers and suppliers of these systems is not entirely suitable to the task and the systems are not interoperable. This indeed is a major obstacle that cannot be solved by pathologists. As long as different microscope manufacturers do not set standards to make their different systems compatible, it is unlikely that telepathology will become a widely used tool in diagnostic pathology. In that case, it may well be that vir- tual microscopy manufacturers will take over this role which in the long term could completely change the landscape in a pathology laboratory.

\section{Acknowledgements}

This study would not have been possible without the enthusiastic help of Dr. Th. Halma (Academic Hospital Vrije Universiteit, Amsterdam) with the logistics and moral support. The following persons at KPN Telecom have been essential for this study: B. van Wijhe, J. Teterissa, R. Jockin and A. Noorbergen. We thank the following pathologists for giving their impressions about the video-conferencing and telepathology aspects of the system: Prof. Dr. P. van der Valk and Dr. J.L.G. Blaauwgeers, Amsterdam; Dr. E. Weltevreden, Apeldoorn, Dr. H.M. Ruitenberg, Utrecht, Prof. Dr. W.J. Mooi, Amsterdam, Dr. J.W. Jansen, Alkmaar/Hoorn, Dr. P.J. Holwerda, Dr. Th.M. van Leeuwen and Dr. M. Jiwa, Alkmaar. We thank José Konneman, Freddy Woltinge, Marc Broeckaert and Jane Brugghe for their skilled technical assistance.

\section{References}

[1] Z. Agha, R.S. Weinstein and B.E. Dunn, Cost minimization analysis of telepathology, Am. J. Clin. Pathol. 112 (1999), 470478.

[2] R.L. Becker, Jr., C.S. Specht, R. Jones, M.E. RuedaPedraza and T.J. O'Leary, Use of a remote video microscopy (telepathology) as an adjunct to neurosurgical frozen section consultation. Hum. Pathol. 24 (1993), 909-911.

[3] K.T. Bird, Telemedicine: concept and practice, in: Telemedicine: Explorations in the use of Telecommunications in Health Care, R.L. Bashshur, P.A. Armstrong and Z.I. Youssef, eds, Charles C. Thomas, Springfield, IL, 1975, pp. 89-112.

[4] P.W. Callas, K.O. Leslie, A.R. Mattia, D.L. Weaver, D. Cook, B. Travis, D.E. Stanley, L.A. Rogers, S.L. Mount, T.D. Trainer, M.A. Zarka and R.M. Belding, Diagnostic accuracy of a rural live video telepathology system, Am. J. Surg. Pathol. 21 (1997), 812-819.

[5] V. Della Mea, P. Cataldi, S. Boi, N. Finato, P. Dalla Palma and C.A. Beltrami, Image sampling in static telepathology for frozen section diagnosis, J. Clin. Pathol. 52 (1999), 761-765.

[6] V. Della Mea, P. Cataldi, B. Pertoldi and C.A. Beltrami, Combining dynamic and static robotic telepathology: a report on 184 consecutive cases of frozen sections, histology and cytology, Anal. Cell. Pathol. 20 (2000), 33-39.

[7] V. Della Mea, D. Cortolezzis and C.A. Beltrami, The economics of telepathology - a case study, J. Telemed. Telecare 6 (Suppl. 1) (2000), S168-169. 
[8] M. Dietel, T.N. Nguyen-Dobinsky and P. Hufnagl, The UICC Telepathology Consultation Center. International Union Against Cancer, A global approach to improving consultation for pathologists in cancer diagnosis, Cancer 89 (2000), 187191.

[9] B.E. Dunn, H. Choi, U.A. Almagro, D.L. Recla, E.A. Krupinski and R.S. Weinstein, Routine surgical telepathology in the department of veterans affairs: experience-related improvement in pathologists performance in 2200 cases, Telemed. J. 5 (1999), 323-337.

[10] L. Gonzalves and C. Cunha, Telemedicine project in the Azores Islands, Arch. Anat. Cytol. Pathol. 43 (1995), 285-287.

[11] K. Kayser and C. Kayser, Telepathology - aspects of social influence and quality control, Elec. J. Pathol. Histol. 3(3) (1996), No. 963-04.

[12] K. Kayser, J. Szymas and R. Weinstein, Telepathology Telecommunication, Electronic Education and Publication in Pathology, Springer, Heidelberg, New York, 1999.

[13] K. Kayser and G. Kayser, Basic aspects of and recent developments in telepathology in Europe, with specific emphasis on quality assurance, Anal. Quant. Cytol. Histol. 21 (1999), 319328.

[14] P. Knudsen, A.J. Ryther, J.A. Nesheim, V.M. Abeler, J.M. Nesland and H.E. Danielsen, Telepathology at the Norwegian Radium Hospital, Tidsskr. Nor. Laegeforen 120 (2000), 21702173.

[15] T. Mairinger, T.T. Netzer, W. Schoner and A. Gschwendtner, Pathologists' attitudes to implementing telepathology, J. Telemed. Telecare 4 (1998), 41-46.

[16] I. Nordum, B. Engum, E. Rinde, A. Finseth, H. Ericssonc, M. Kearney, H. Stalsberg and T.J. Eide, Remote frozen section service: a telepathology project in northern Norway, Hum. Pathol. 22 (1991), 514-518.
[17] M. Oberholzer, H.R. Fischer, H. Christen, S. Gerber, M. Bruehlmann, M. Mihatsch, M. Famos, C. Winkler, P. Fehr, L. Baechtold and K. Kayser, Telepathology with ISDN - A new tool for image transfer in surgical pathology, Hum. Pathol. 24 (1993), 1078-1085.

[18] M. Oberholzer, H.R. Fischer, H. Christen, S. Gerber, M. Bruhlmann, M.J. Mihatsch, T. Gahm, M. Famos, C. Winkler and P. Fehr, Telepathology: frozen section diagnosis at a distance, Virchows Arch. 426 (1995), 3-9.

[19] S. Olsson and C. Busch, A national telepathology trial in Sweden: feasibility and assessment, Arch. Anat. Cytol. Pathol. $\mathbf{4 3}$ (1995), 234-241.

[20] J.H. Saltz, Digital pathology - The big picture. Editorial, Hum. Pathol. 31 (2000), 779-780.

[21] R.P. Singson, S. Natarajan, J.K. Greenson and A.M. Marchevsky, Virtual microscopy and the Internet as telepathology consultation tools. A study of gastrointestinal biopsy specimens, Am. J. Clin. Pathol. 111 (1999), 792-795.

[22] C. Sowter and C.A. Wells, Telepathology: assessment of the implications and applications of telepathology for practical diagnostic pathology, J. Clin. Pathol. 51 (1998), 714-715.

[23] D.S. Weinberg, F.A. Allaert, P. Dussere, F. Drouot, B. Retalliau, W.R. Welch, J. Longtine, G. Brodsky, R. Folkerth and M. Doolittle, Telepathology diagnosis by means of digital still images: an international validation study, Hum. Pathol. 27 (1996), 111-118.

[24] R.S. Weinstein, Editorial: Telepathology comes of age in Norway, Hum. Pathol. 22 (1991), 511-513.

[25] R.S. Weinstein, Editorial: Static image telepathology in perspective, Hum. Pathol. 27 (1996), 99-101.

[26] C.A. Wells and C. Sowter, Telepathology: a diagnostic tool for the millennium?, J. Pathol. 191 (2000), 1-7. 


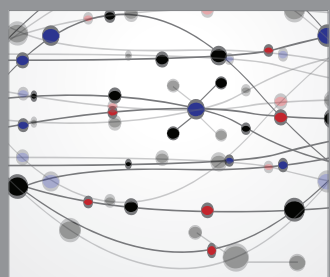

The Scientific World Journal
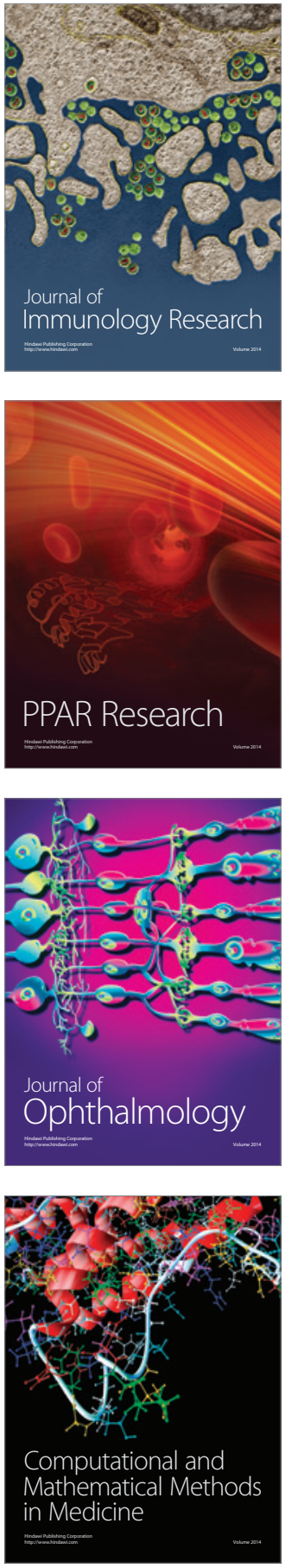

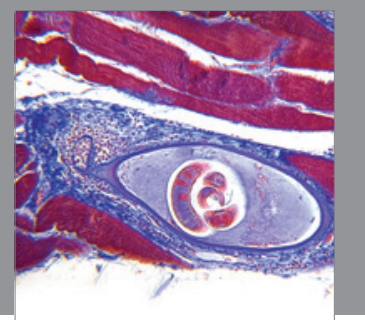

Gastroenterology

Research and Practice
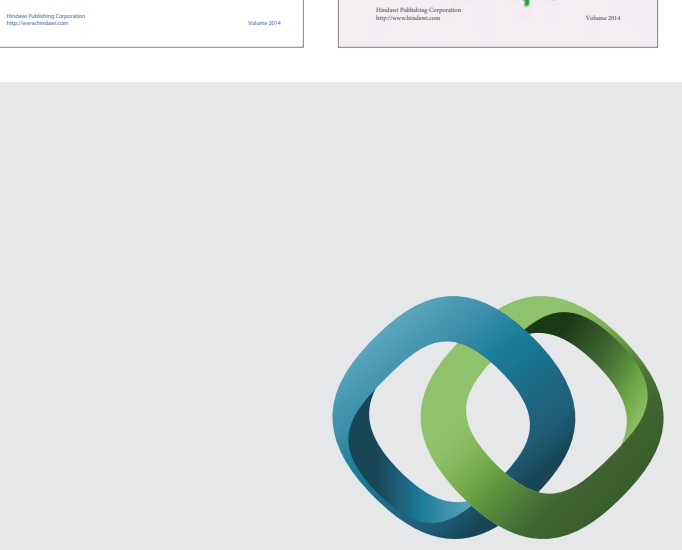

\section{Hindawi}

Submit your manuscripts at

http://www.hindawi.com
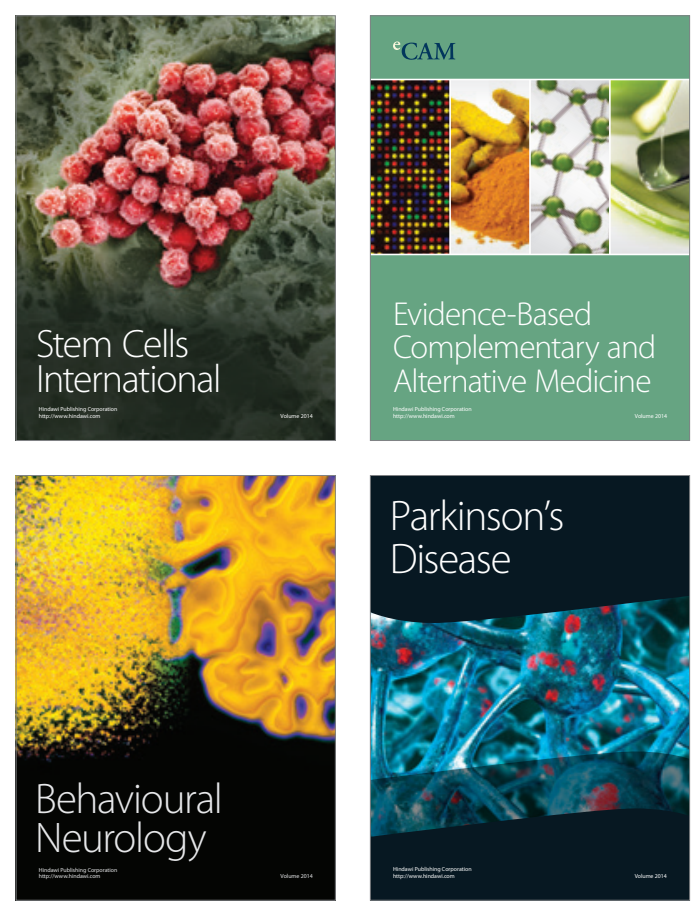

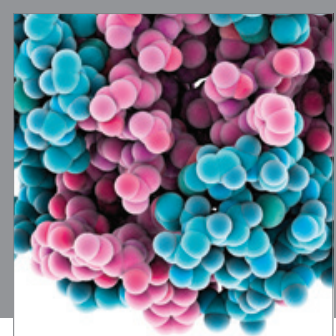

Journal of
Diabetes Research

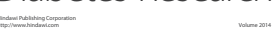

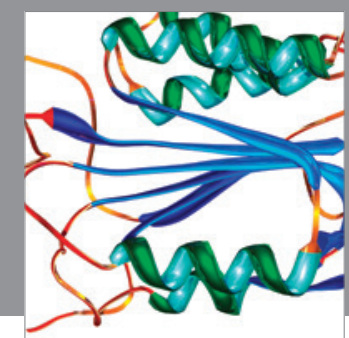

Disease Markers
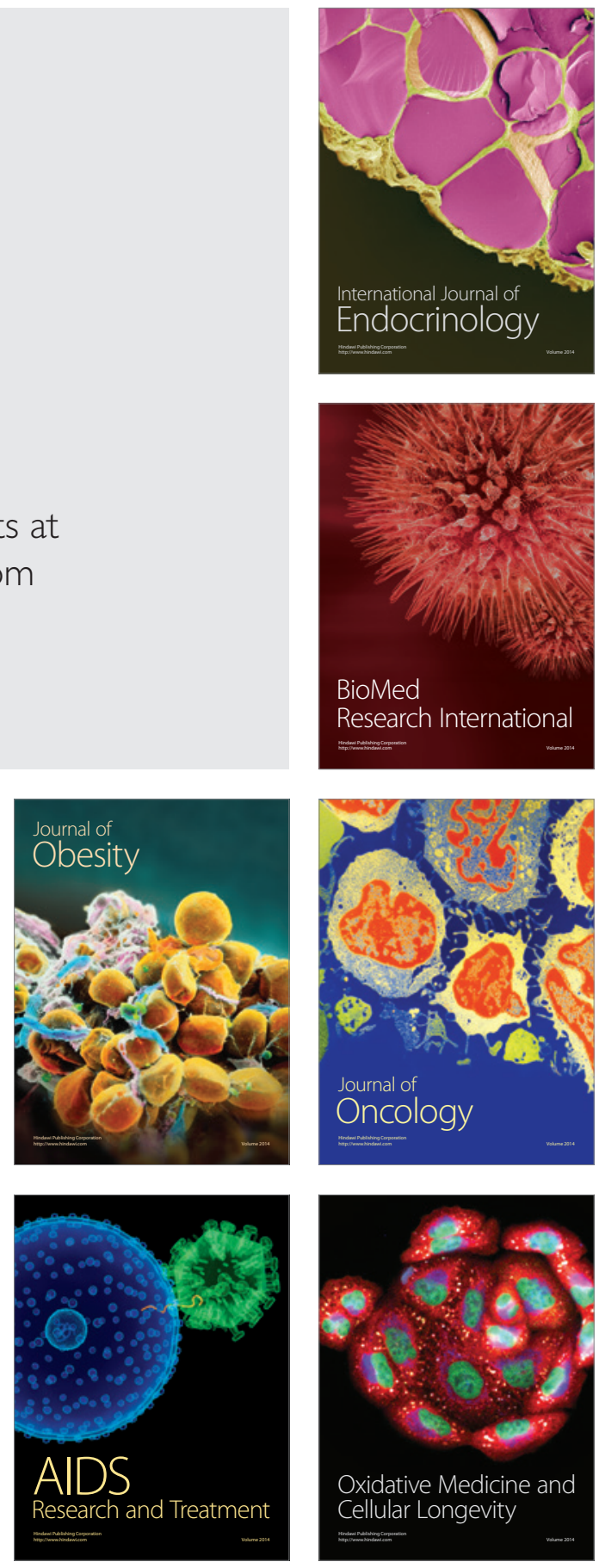